\title{
The Anatomy and Anatomical Variations of the Round Window Prechamber and Their Implications on Cochlear Implantation: An Anatomical, Imaging, and Surgical Study
}

\author{
Ahmed Mohamed Mehanna ${ }^{1}$ (1) Moustafa Mohamed Abdelnaby ${ }^{1}$ Mohamed Eid² \\ ${ }^{1}$ Department of Otolaryngology, Alexandria University, Midan al \\ Khartoum, Alexandria, Egypt \\ ${ }^{2}$ Department of Radiodiagnosis, Faculty of Medicine of Alexandria \\ University, Midan al Khartoum, Alexandria, Egypt \\ Address for correspondence Dr. Ahmed Mohamed Mehanna, \\ Department of Otolaryngology, Alexandria University, Midan al \\ Khartoum, Alexandria, Egypt 11511 (e-mail: mehanna81@hotmail.com).
}

Int Arch Otorhinolaryngol 2020;24(3):e288-e298.

\begin{abstract}
Keywords

- Cochlear Implantation

- facial nerve

- middle ear

- cochlea

Introduction Over the last decades, there has been a tremendous increase in the number of cochlear implant recipients and, consequently, there is a recent increase of interest in the proper understanding of the anatomy of the round window (RW), which is the most important anatomical land mark during cochlear implant surgery.

Objectives The present study was undertaken to assess the detailed surgical and radiological anatomy of the RW prechamber; its shape, directions, measurements, common anatomic variations, and its relationships with different surrounding structures as related to cochlear implantation.

Methods A total of 20 cadaveric specimens of human temporal bone were microscopically dissected for the anatomical assessment of the measurements of the RW and its relation to surrounding structures in the tympanum. A total of 20 patients were subjected to cochlear implantation, and a radiological and surgical assessment of the anatomy of their RW prechambers was performed.

Results The distances between the RW and the facial canal (FC), the jugular fossa (JF), the carotid canal (CC), and the oval window (OW) were measured. Among the cases subjected to cochlear implantation, the infracochlear tunnel was studied radiologically; the lengths of the anterior and posterior pillars were assessed, and the relation with the direction at which the RW faces was statistically analyzed.

Conclusions Proper understanding of the topographic anatomy of the RW, including its direction of opening and the distances from different adjacent structures in the tympanum, is essential for a successful cochlear implantation surgery, since it can help decision-making before the surgery and is useful to avoid many complications, such as misplaced electrode and iatrogenic injury to the surrounding structures.
\end{abstract}

received

November 23, 2018

accepted

September 7, 2019

published online

December 13, 2019
DOI https://doi.org/

10.1055/s-0039-1698783.

ISSN 1809-9777.
Copyright $\odot 2020$ by Thieme Revinter

Publicações Ltda, Rio de Janeiro, Brazil
License terms

c) $9 \ominus \$$ 


\section{Introduction}

Over the last decades, there has been a tremendous increase in the number of cochlear implant recipients, and, consequently, there is a recent increase of interest in the proper understanding of the anatomy of the round window (RW), which is the most important anatomical landmark, not only in cochlear implant surgery but also in other otologic procedures. A thorough and proper understanding of the anatomy of the RW is now considered essential and mandatory for the practicing otolaryngologist rather than for an experimental scientist. ${ }^{1}$

The round window membrane (RWM) is normally hidden under a boney overhang, termed the RW niche (RWN), which is formed by a posterior pillar (postis posterior), the tegmen, and the anterior pillar (postis anterior). The RWM with its niche is termed the RW prechamber. The membrane itself is located at the end of the scala tympani anterolateral to the hook region. ${ }^{2}$

Insertion of the electrode array either through the RWM or by means of creation of a cochleostomy has been debated for a long time a; however, both approaches depend on the meticulous identification of the RW. The early reports of cochlear implantation emphasized insertion through the RWM; this approach was replaced by drilling a cochleostomy anteroinferior to the RWM. The crista fenestra, which is preserved in the first approach, usually obscures the vision of the surgeon during RW insertion. Recent researches emphasized the residual hearing preservation and a soft atraumatic surgery. Round window membrane insertion with preservation of the crista fenestra is now the insertion site of choice for many surgeons, as it is claimed that the electrode will be kept in place by this bone; therefore, if the electrode is inserted through the cochleostomy, the point of first contact to the basilar membrane is $5 \mathrm{~mm}$ far anterior, so the contact length toward the modiolus is $5 \mathrm{~mm}$ shorter. $^{3-5}$

The position of the RWN, its shape, and the direction that its opening faces are variable among individuals. The awareness of possible anatomical variations of the RW, as it relates to the different anatomical structures in the tympanum, can help with the decision-making process before surgery regarding the type, the length, and the site of insertion of the cochlear implant electrode array and it, subsequently, minimizes the rate of complications and misplacement. ${ }^{6,7}$

\section{Materials and Methods}

We performed a prospective study on 20 cadaveric specimens and on 20 patients subjected to cochlear implantation at the main university hospital in Egypt, to assess the detailed surgical and radiological anatomy of the RW prechamber; its shape and direction, measurements, common anatomic variations, and the relationships with the different surrounding structures as related to cochlear implantation. The present study was performed after approval from the ethical committee for research at the university hospital and from the affiliated hospitals.
A) A total of 20 cadaveric specimens of human temporal bones with no available data about name and sex were fixed in $10 \%$ formalin and then were microscopically dissected as following: cortical mastoidectomy was performed, followed by posterior tympanotomy, then the tympanic membrane and the posterior meatal skin were removed, for assessment of the following parameters:

\section{I) Round window morphology:}

1) RW visibility through the posterior tympanotomy and through the external auditory canal.

2) RW shape; oval, rounded, triangular, pear-shaped or quadrangular.

3) The direction of the RW opening; posterior, if it faces completely to the posterior mesotympanum; inferior, if it faces completely down; and, lastly, posterinferior if between both sites.

4) The presence or absence of a well-defined infracochlear air cell track.

5) In all the dissected temporal bones, full visualization of the RWM was achieved after complete removal of the bony overhangs. The crista fenestra was properly identified as a sharp bony crest located in the anterior and inferior borders of the niche with variable shapes and variable degrees of narrowing to the access of the scala tympani of the basal turn of the cochlea.

II) Round window measurements:

The dissected bones were photographed with a millimeter scale using a Nikon D3200 camera (Nikon, Tokyo, Japan). Then the photographs were imported to a computer, and by using a special software J1.46r software (National Institute of Mental Health, Bethesda, Maryland, USA) after proper calibration and at $5 \mathrm{x}$ magnification, the following parameters were measured and scaled from pixel-to-millimeter conversion.

1) Maximum height of the RW (RWh).

2) Maximum width of the RW (RWw).

3) Minimum distance between the RW and the mastoid segment of the facial nerve canal (FC) (RW-FC).

4) Minimum distance between the RW and the roof of the jugular fossa (JF) (RW-JF).

5) Minimum distance between the RW and the oval window (OW) (RW-OW).

6) Minimum distance between the RW and the carotid canal (CC) (RW-CC).

The sample size in the temporal bone study was calculated based on previous studies and by using the MedCalc statistical software (MedCalc, Ostend, Belgium) and was defined as at least 19 temporal bones when compared with other studies in the literature. The area under the receiver operating characteristic (ROC) curve was assumed to be 0.8 , a $\alpha$ of 0.05 , and a power of study of $80 \%$. 
B) A total of 20 patients were subjected to cochlear implantation at the main university hospital in Egypt. The anatomy of the RW was assessed radiologically before the surgery, and was also evaluated during the surgery.

1) Radiological assessment:

A) The maximum transverse diameter of the RW.

B) The depth of the RWN. The distance between the middle of the operculum width and the deepest part of the RWN.

C) The pattern of pneumatization of the infracochlear air cell track (subcochlear canaliculus).

All of the imaging examinations were performed with a SOMATOM Definition AS 20-section multidetector row CT scanner (Siemens, Munich, Germany). A section thickness of $0.5 \mathrm{~mm}$, spacing of $0.3 \mathrm{~mm}$ with overlap, $\mathrm{mA}$ of 250 milliseconds, $\mathrm{kV}$ of 120 milliseconds, helical pitch of 0.625 , rotation time of 0.8 second, and field of view of $240 \mathrm{~mm}$ were adopted. The raw data were reconstructed by using a bone algorithm to provide optimal visualization of the bony anatomy of the temporal bone. The images of the temporal bone were displayed at a window center of $400 \mathrm{HU}$ and at a window width of 4,000 $\mathrm{HU}$.

The new OTOPLAN otological tablet based planning platform (Cascination AG, Bern, Switzerland) was used in all cases subjected to cochlear implantation, which can quickly generate patient-specific 3D reconstruction from medical images and easily visualizes the unique anatomy of each patient.

2) Round window morphology assessment during surgery:

A) The length of both the posterior pillar and the anterior pillar.

B) The direction that the RW faces, whether posterior, inferior, or posteroinferior.

\section{Results}

\section{Round Window Morphology and Parameters}

In the current study, the niche was visible through the external auditory canal in 14 specimens (70\%), and was visible in 16 out of the 20 specimens through the facial recess (80\%). The RW exhibited different shapes; we found that an oval RW was the most commonly encountered, as $50 \%$ of the specimens had an oval RWN, followed by round (25\%), triangular (10\%), pear-shaped (5\%), and, lastly, quadrangular in only 2 specimens $(10 \%)$ ( - Table $\mathbf{1}$ ).

The direction that the niche faced was posteroinferior in $50 \%$ of the specimens, and, in $40 \%$, it faced inferiorly, while in only $10 \%$ the direction was posterior. The lateral opening of the infracochlear air cell track was clearly visible in $60 \%$ of the studied specimens, it was barely visible in $10 \%$ of the cases; only a small hole was detected under the finiculus inferior to the RW in $30 \%$ of the studied specimens, and lastly, in 2 specimens, its lateral end was not found and the finiculus bone merged medially with the fustis.

\section{Round Window Measurements}

The mean maximum height of the round window niche was $1.53 \pm 0.41 \mathrm{~mm}$, ranging from 0.9 to $2.3 \mathrm{~mm} ; 75 \%$ of the specimens measured between 1 and $2 \mathrm{~mm}$, while $15 \%$ measured $>2 \mathrm{~mm}$, and $10 \%$ measured $<1 \mathrm{~mm}$. We found that the RW height gives an idea about the depth of the RW prechamber.

The mean width of the RW was $1.18 \pm 0.25 \mathrm{~mm}$, ranging from 0.8 to $1.65 \mathrm{~mm}$. A total of $75 \%$ of the specimens measured $>1 \mathrm{~mm}$, and $25 \%$ measured $<1 \mathrm{~mm}$.

The surface area of the RWN was determined by measuring the circumference of the niche after the excision of the RWM in square millimeters; this surface area was $1.52 \pm 0.28 \mathrm{~mm}^{2}$, ranging from 1.24 to $1.52 \mathrm{~mm}^{2}$.

In our study, we assessed the minimal distance between the RW niche and the surrounding anatomical structures. The results were as follows: the mean minimal RW-OW distance was $2.44 \pm 0.42 \mathrm{~mm}$, ranging from 1.7 to $3.05 \mathrm{~mm}$; the mean minimal RW-CC distance was $7.9 \pm 1.44 \mathrm{~mm}$, ranging from 5.3 to $11.05 \mathrm{~mm}$; a total of $5 \%$ measured $<6$ $\mathrm{mm}, 75 \%$ measured between 6 and $8 \mathrm{~mm}$, and $5 \%$ measured $>8 \mathrm{~mm}$. The mean minimal RW-JF distance was $2.77 \pm 0.42 \mathrm{~mm}$, ranging from 1.95 to $3.9 \mathrm{~mm}$; a total of $5 \%$ measured $<2 \mathrm{~mm}, 70 \%$ measured between 2 and $3 \mathrm{~mm}$, and $25 \%$ measured $>3 \mathrm{~mm}$. Lastly, we measured the minimal RW-FC distance, which measured $5.55 \pm 1.18 \mathrm{~mm}$, ranging from 3.25 to $7.75 \mathrm{~mm}$. A total of $15 \%$ measured $<4$ $\mathrm{mm}, 50 \%$ measured between 4 and $6 \mathrm{~mm}$, and 35\% measured $>6 \mathrm{~mm}$. (-Fig. 1)

The crista fenestra was clearly identified in 18 specimens out of the 20 dissected temporal bones; its shape was rectangular, causing significant narrowing of the RW region and subsequently to cochlear implant electrode insertion among 6 temporal bones, and showed semilunar shape in 7 cadavers, triangular in shape among 3 cases, and, lastly, it was rudimentary in 2 temporal bones. (-Fig. 2 )

\section{Radiological Assessment}

The mean maximum transverse diameter of the round window niche was measured in the axial and coronal plane of the high-resolution computed tomography (HRCT) scan; it measured $1.27 \pm 0.4 \mathrm{~mm}$ with a range between 1.2 and $2.3 \mathrm{~mm}$. The height of the RW prechamber (the depth of the prechamber) was measured in the sagittal plane, and it measured $1.52 \pm 0.38 \mathrm{~mm}$ with a range between 1.1 and $2.4 \mathrm{~mm}$.

Serial sections in the sagittal view using the OTOPLAN otological tablet based planning platform was used in cases prior to cochlear implantation. It can generate patient-specific 3D reconstruction from ordinary medical images and visualizes the unique anatomy of each patient, including the optimal electrode array for each candidate. This software enables the surgeon to achieve proper visualization of the cochlear anatomy, including the RW and its relations with the surrounding anatomical structures prior to the surgery. In the current study, imaging data was transformed to this software for assessment of the infracochlear air cell track pneumatization. - Fig. 3 

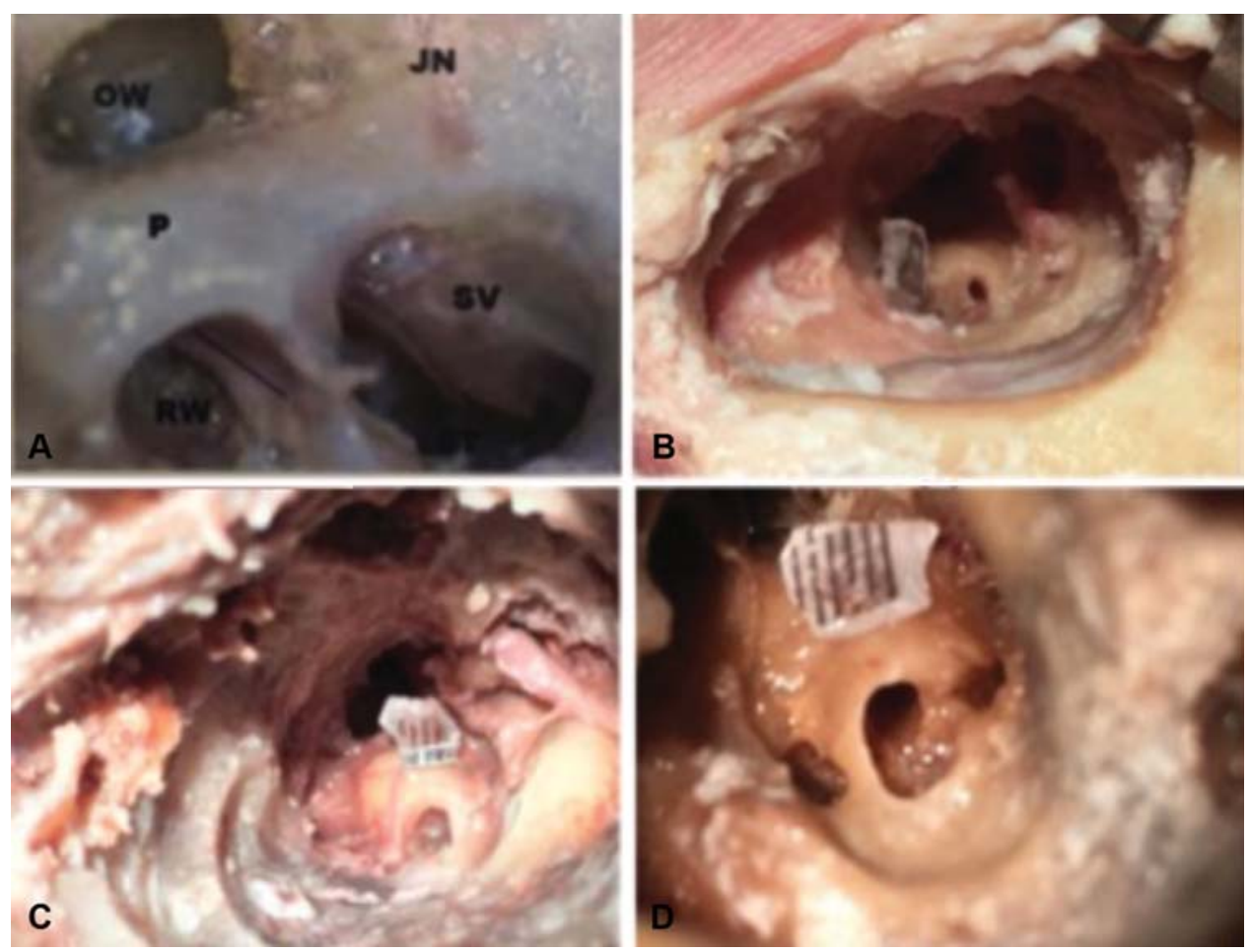

Fig. 1 Microscopic view illustrating the measurement of the minimum distance between (A) round window and oval window, (B) round window and internal carotid, (C) round window and jugular fossa, (D) round window and the vertical segment of the facial nerve.
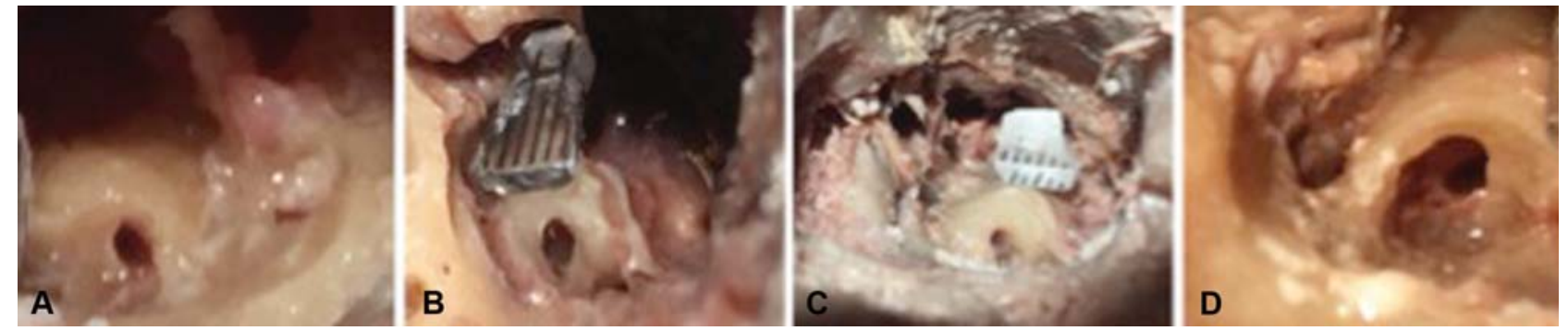

Fig. 2 Different shapes of the crista fenestra. (A) Absent, (B) Rudimentary, (C) Rectangular obstructing most of the round window region, (D) Semilunar in shape.

Four different types of pneumatization of the infracochlear air cell track were found, and, subsequently, we classified the subcochlear canaliculus in our study into:

Type 1: Poorly pneumatized infracochlear air cell tract (-Fig. 3A). This was noticed in only 3 cases.

Type 2: Well-pneumatized but not reaching the petrous apex (-Fig. 3B), and this type was found in 6 cases.

Type 3: Well-pneumatized reaching the petrous apex, no connection with the petrous apex air cells (-Fig. 3C); this type was the most common type in our study, and was found in 9 cases.

Type 4: Well-pneumatized and connected to the petrous apex air cells ( - Fig. 3D); only 2 cases had this type of pneumatization.

In two cases, the cochlear implant electrode was misplaced through this tunnel to an extracochlear site, one into the petrous apex (Type 4 infracochlear tunnel), while in the other case the electrode was found in the hypotympanum inferior to the cochlea (Type3 infracochlear tunnel). Both cases have been excluded from the study. (-Fig. 4)

\section{Surgical Assessment}

The surgical anatomy of the RWN was evaluated in 20 patients subjected to cochlear implantation; the endoscopic assisted suprameatal approach was used in 9 cases. The Advanced Image and Data Acquisition (AIDA) system with a high definition Storz endoscopic camera (Karl Storz, Tuttlingen, Germany) (Image1) was attached to either the surgical microscope or directly to the endoscope using $0^{\circ}$ and $30^{\circ}$ rigid Hopkins rod telescopes with a $3 \mathrm{~mm}$ outside diameter, $15 \mathrm{~cm}$ length, (Karl Storz, Tuttlingen, Germany).

A) The direction of the RWN; the RW was found facing posteroinferior in 11 cases (55\%), in 7 cases the RW 

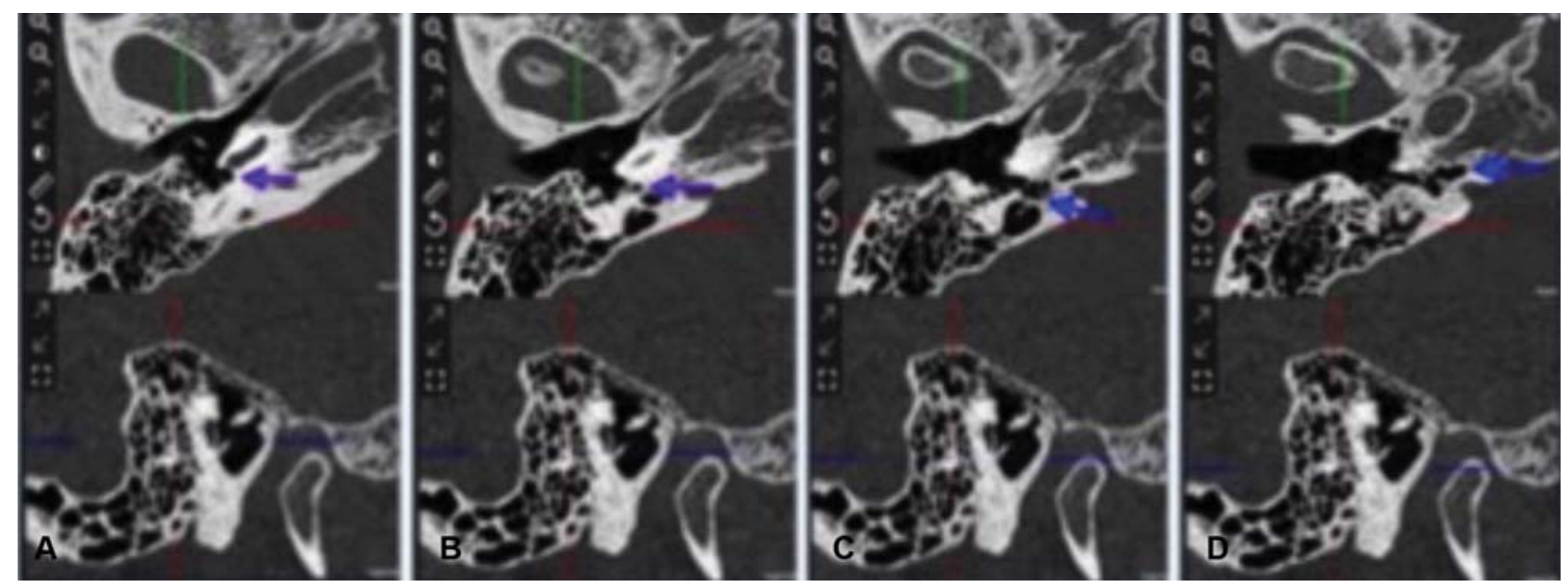

Fig. 3 Serial sections in the sagittal view using the MED-EL otological tablet based planning platform (OTOPLAN) showing the degree of pneumatization of the infracochlear tunnel. (A, B) the pneumatization is limited just inferior to the cochlea, $(C, D)$ the pneumatization reaches far anterior until the petrous apex.
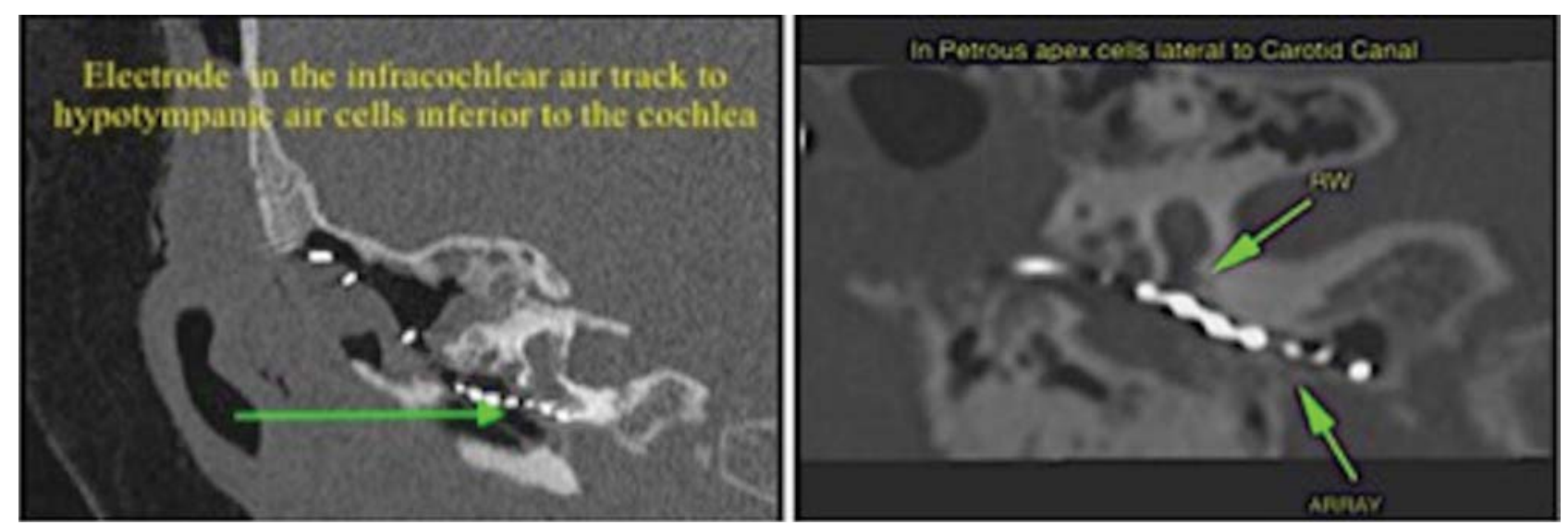

Fig. 4 Two cases of misplaced cochlear implant electrode not included in our study, in which the cochlear implant electrode passed through the subcochlear canaliculus into an extracochlear site.

direction was inferior, and, lastly, in only 2 cases (10\%), the direction was posterior. (-Fig.5)

B) The length of the anterior and posterior pillars were measured ( - Table 4 ), the photographs were imported to a computer, and by using a special software (Image J1.46r software [National Institute of Mental Health, Bethesda, Maryland, USA]) after proper calibration and at 5x magnification, they were used to assess:

1) The postis anterior length (the anterior pillar), from the dome (the most superior point) of the RW tegmen until

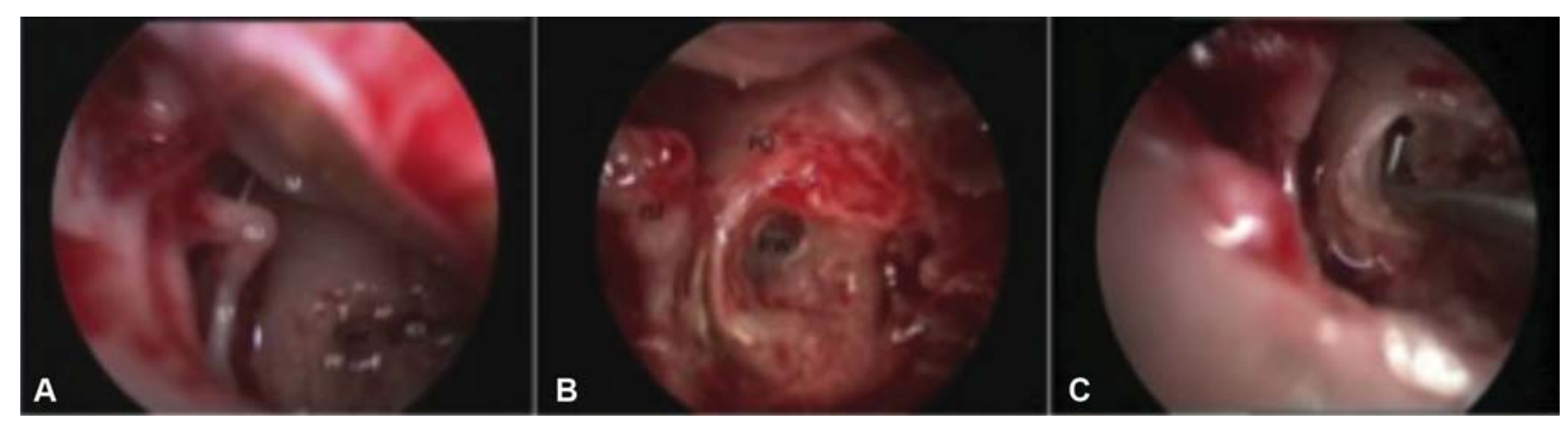

Fig. 5 The round window prechamber during endoscopic assisted suprameatal approach for cochlear implantation showing the direction at which the RW faces according to the relation between the postis posterior and the postis anterior. (A) It faces posteroinferior, both pillars are equal in length, (B) It faces posterior, the anterior pillar is much longer than the posterior, C, it faces inferior, the posterior pillar is much longer than the anterior. 
Table 1 Summary of the round window visibility, shape, direction, and the crista fenestra among the 20 cadaveric specimens

\begin{tabular}{|c|c|c|c|c|c|c|}
\hline & $\begin{array}{l}\text { Visibility } \\
\text { through FR }\end{array}$ & Visibility through EAC & RW shape & Direction of opening & Crista fenestra & Infracochlear tunnel \\
\hline 1 & Visible & Not Visible & Pear shape & Posteroinferior & Rectangular & Clearly visible \\
\hline 2 & Visible & Visible & Rounded & Posteroinferior & Rectangular & Clearly visible \\
\hline 3 & Visible & Visible & Rounded & Inferior & Rudimentary & Clearly visible \\
\hline 4 & Visible & Visible & Pear shape & Posteroinferior & Semilunar & Clearly visible \\
\hline 5 & Visible & Visible & Rounded & Inferior & Triangular & Barely seen \\
\hline 6 & Not Visible & Not Visible & Triangular & Posterior & Semilunar & Barely seen \\
\hline 7 & Visible & Visible & Oval & Inferior & Rectangular & Barely seen \\
\hline 8 & Visible & Visible & Oval & Inferior & Triangular & Clearly visible \\
\hline 9 & Not Visible & Visible & Oval & Posterior & Semilunar & Barely seen \\
\hline 10 & Visible & Not Visible & Rounded & Inferior & Rectangular & Barely seen \\
\hline 11 & Visible & Visible & Oval & Posteroinferior & Semilunar & Barely seen \\
\hline 12 & Not visible & Visible & Oval & Posteroinferior & Absent & Clearly visible \\
\hline 13 & Visible & Visible & Oval & Inferior & Absent & Clearly visible \\
\hline 14 & Visible & Not Visible & Triangular & Posteroinferior & Semilunar & Invisible \\
\hline 15 & Visible & Visible & Oval & Posteroinferior & Triangular & Clearly visible \\
\hline 16 & Not Visible & Visible & Oval & Posteroinferior & Rectangular & Clearly visible \\
\hline 17 & Visible & Not Visible & Rounded & Inferior & Semilunar & Invisible \\
\hline 18 & Visible & Visible & Oval & Posteroinferior & Rudimentary & Clearly visible \\
\hline 19 & Visible & Visible & Oval & Inferior & Semilunar & Clearly visible \\
\hline 20 & Visible & Not Visible & Quadrangular & Posteroinferior & Rectangular & Clearly visible \\
\hline
\end{tabular}

Abbreviations: EAC, external auditory canal; FR, facial recess; RW, round window.

Table 2 Summarizes the statistical data for the measurements of both the postis anterior and posterior of the round window prechamber in millimeters

\begin{tabular}{|l|l|l|}
\hline$(\boldsymbol{n}=\mathbf{2 0})$ & $\begin{array}{l}\text { Anterior pillar } \\
\text { length }\end{array}$ & $\begin{array}{l}\text { Posterior pillar } \\
\text { length }\end{array}$ \\
\hline Mean \pm SD & $2.22 \pm 0.18$ & $2.09 \pm 0.20$ \\
\hline Median & 2.3 & 2.17 \\
\hline $\begin{array}{l}\text { Minimum- } \\
\text { Maximum }\end{array}$ & $1.97-2.64$ & $1.70-2.37$ \\
\hline Range & 0.63 & 0.67 \\
\hline
\end{tabular}

Abbreviation: SD, standard deviation.

its end where it meets the fustis of the RW prechamber (a smooth bony structure, which forms the floor of the RW prechamber indicating the entrance to the round window membrane). (-Fig. 5)

2) The postis posterior length (the posterior pillar), from the dome (the most superior point) of the RW tegmen until its beginning (the junction with the fustis). (-Fig. 5)

Statistical analysis of the relation between the length of both pillars and the direction at which the RW faces was done using IBM SPSS Statistics for Windows, Version 20.0 (IBM Corp., Armonk, NY, USA), the difference in length between the anterior and posterior pillars (ante- rior pillar length - posterior pillar length) was calculated in $\mathrm{mm}$.

- Tables 2, 3, 4 show a significant difference among the three directions of the RW (posteroinferior, inferior, and posterior) in relation to the difference in length between the anterior and posterior pillar. If the mean difference between both pillars was $(0.02 \pm 0.03)$; both pillars are almost equal, the RW was facing postero-inferior, and this was found in 11 cases. The direction of the RW was inferior in 7 cases when the mean difference was $(-0.41 \pm 0.06)$; the posterior pillar was significantly longer than the anterior one. Lastly posteriorly facing RW niche was found only in two cases and the difference between both pillars was $(0.61 \pm 0.01)$; Significantly longer anterior pillar. (-Fig. 4)

\section{Discussion}

The RW has been a subject of anatomical interest ever since it was discovered and named by Gabriel Fallopius in the $16^{\text {th }}$ century. The RW prechamber is a three-dimensional space lying between the RWN and the RW membrane. It is funnelshaped or conical, becoming narrower toward its fundus, laterally toward the middle ear is the RWN, and it ends medially by the round membrane. ${ }^{8}$

In the current work, a prospective study on 20 cadaveric specimens and on 20 patients subjected to cochlear implantation at the main university hospital was 
Table 3 Distribution of the studied round window directions according to difference in length between anterior and posterior pillars

\begin{tabular}{|l|l|l|l|l|}
\hline Direction of cord & $\begin{array}{l}\text { Posteroinferior } \\
(\boldsymbol{n}=\mathbf{1 1})\end{array}$ & $\begin{array}{l}\text { Inferior } \\
(\boldsymbol{n}=\mathbf{7})\end{array}$ & $\begin{array}{l}\text { Posterior } \\
(\boldsymbol{n}=\mathbf{2})\end{array}$ & Kruskal-Wallis test \\
\hline Mean \pm SD & $0.02 \pm 0.03$ & $-0.41 \pm 0.06$ & $0.61 \pm 0.01$ & $X^{2}=15.58$ \\
$p<0.001^{*}$ & \\
\hline Median & 0.0 & -0.42 & 0.61 & $0.60-0.62$ \\
\hline Minimum-Maximum & $0.00-0.10$ & $-0.49-0.30$ & 0.02 & \\
\hline Range & 0.10 & 0.19 & & \\
\hline
\end{tabular}

Abbreviation: SD, standard deviation.

Table 4 Distribution of studied round window directions according to the length of the anterior and of the posterior pillar

\begin{tabular}{|c|c|c|c|}
\hline Direction of cord & $\begin{array}{l}\text { Posteroinferior } \\
(n=11)\end{array}$ & $\begin{array}{l}\text { Inferior } \\
(n=7)\end{array}$ & $\begin{array}{l}\text { Posterior } \\
(n=2)\end{array}$ \\
\hline \multicolumn{4}{|l|}{ Anterior pillar length } \\
\hline Mean \pm SD & $2.07 \pm 0.15$ & $1.85 \pm 0.07$ & $2.35 \pm 0.07$ \\
\hline Median & 2.11 & 1.85 & 2.35 \\
\hline Minimum-Maximum & $1.80-2.35$ & $1.77-1.95$ & $2.30-2.40$ \\
\hline Range & 0.55 & 0.18 & 0.10 \\
\hline \multicolumn{4}{|l|}{ Posterior pillar length } \\
\hline Mean \pm SD & $2.05 \pm 0.16$ & $2.27 \pm 0.06$ & $1.74 \pm 0.06$ \\
\hline Median & 2.12 & 2.25 & 1.74 \\
\hline Minimum-Maximum & $1.80-2.30$ & $2.19-2.37$ & $1.70-1.74$ \\
\hline Range & 0.50 & 0.18 & 0.08 \\
\hline
\end{tabular}

conducted to assess the detailed surgical and radiological anatomy of the RW prechamber relevant for cochlear implantation.

\section{A) Anatomical temporal bone study:}

In our collection, among the 20 cadaveric specimens, different shapes were identified; the oval RW was the most commonly encountered. Our results were similar to that of a previous study conducted by Singla et al on 50 gross cadaveric temporal bones. In another study done by Tóth et al, they reported other RW shapes, such as comma shape and pinpoint ones, and they also emphasized that age does not influence the shape of the RW. ${ }^{9-11}$

The RWN is often directly seen within the tympanic cavity after tympanomeatal flap elevation; however, this is not valid in every case. In the current study, it was visible through the external auditory canal in 14 specimens, and was visible in 16 out of the 20 specimens through the FR. These results were consistent with the results concluded by Hamamoto et al using 22 temporal bones; in $77 \%$ of the temporal bones, the RWN was visible through posterior tympanostomy; on the other hand, the results were slightly different from those of Goravalingappa, who reported $40 \%$ of invisible RWNs through posterior tympanostomy. The slight variability between our results and those in literature might be attributed to the number of temporal bones used in each study. ${ }^{12,13}$
Among the 20 cadaveric specimens, the direction at which the niche faced was posteroinferior in $50 \%$ of the specimens, and in $40 \%$ it faced inferiorly, while in only $10 \%$ the direction was posterior. These results were consistent with the results documented by Aslan on 11 dry temporal bones and in 9 temporal bone specimens preserved in formalin; they reported that the direction of the niche opening was posterior in $3(15 \%)$, inferior in $9(45 \%)$, and posteroinferior in 8 $(40 \%)^{14}$

Regarding the RW dimensions, measurement parameters were very variable in the literature, Takahashi et al measured the diameter or half diameter of the RW, other authors, as Su et al, and Cohen et al measured its length or width. In our study, owing to the great variability of the RW shape, we preferred to measure the maximum height and width of the RWN. An extremely narrow RW makes the insertion of the electrode array difficult and usually necessitates drilling of the anteroinferior margin of the niche. This area is termed the crista fenestra, which is an obstacle to the insertion of the electrode array to the scala tympani of the basal turn of the cochlea. This drilling allows adjustment of the vector of insertion of the electrode array into the scala tympani; however, it might be not only potentially hazardous because of its close proximity to the hook region, but it might also be traumatic to the cochlea, leading to loss of the residual hearing in hearingpreservation protocols. $^{15-17}$ 
Previous authors, such as Stewart et al, Su et al, Takahashi et al, and Singla et al have measured the RWw, which were $1.5 \mathrm{~mm}, 1.66 \mathrm{~mm}, 2.98 \pm 0.23 \mathrm{~mm}$, and $1.15 \pm 0.39 \mathrm{~mm}$, respectively; in the present work, the mean RWw was $1.18 \pm 0.25 \mathrm{~mm}$. The present results are consistent with Stewart et al, Su et al, and Singla et al. results, but were different from those of Takahashi et al. This can be attributed to the fewer number of cases in their study work, which were only 5 specimens. Regarding RWh, many authors have measured the RWh, as Stewart et al, Cohen et al, and Singla et al; their results were $1.2 \mathrm{~mm}, 1.665 \pm 0.258 \mathrm{~mm}$ and $1.62 \pm 0.77 \mathrm{~mm}$, respectively; in the present study, the mean RWh was $1.53 \pm 0.41 \mathrm{~mm} .^{9,15,16,18}$

The anatomical location of the vertical segment of the FN is at risk of damage during cochlear implantation, especially during posterior tympanotomy. Wide variability in the distance between the RW and the vertical FC is usually present. In the present work, again owing to the wide variability of the shape of the RW, the minimal distance between both of them was measured. The mean minimal distance was $5.55 \pm 1.18 \mathrm{~mm}$. Our results were different from those of Singla et al, who reported the mean distance between the FC and the RW to be $4.28 \pm 0.67 \mathrm{~mm}$, and this might be due to the larger number of cases in their study ( 50 cases) and wider age range (2.5-70 years old). ${ }^{9,19-21}$

Jugular bulb injury during cochlear implantation is a reported complication, especially in high dehiscent bulb. In the present work, the mean minimal RW-JF distance was $2.77 \pm 0.42 \mathrm{~mm}$. Our results were not too far from those of many other authors in the literature; Duan et al, in a study on the computed tomography (CT) scans of 15 normal head specimens, reported the mean RW-JF distance to be $2.10 \mathrm{~mm}$ on the right side and $2.39 \mathrm{~mm}$ on the left side; and our results are also in line with another study conducted by Singla et al, who reported the RW-JF distance as measuring $2.98 \pm 0.68 \mathrm{~mm}$. The study by Stewart et al conducted on 12 vertically sectioned temporal bones, reported the mean distance between the inferior margin of the RWN to the jugular bulb to be $4.4 \mathrm{~mm}$, ranging from 1.4 to $5.7 \mathrm{~mm}$; these measurements were different from those in the current study, the wide variability was attributed to the parameters of measurement and the number of specimens. ${ }^{9,15,22}$

During cochleostomy, anteroinferior to the RW, the knowledge of the precise safe distance of drilling is essential to avoid injury to the internal carotid artery (ICA), as this injury could be potentially fatal. The mean minimal RW-CC distance in the present work was $7.9 \pm 1.44 \mathrm{~mm}$; our results are in agreement with the results of the study by Wysocki et al conducted on 100 temporal bones; they found that the mean RW-CC distance was $8.08 \pm 1.55 \mathrm{~mm}$, and are also in agreement with the results of Singla et al, who stated that the mean RW-CC distance was $8.03 \pm 1.55 \mathrm{~mm}^{9,10}$

According to the present work results, the mean minimal RW-OW distance was $2.44 \pm 0.42 \mathrm{~mm}$; this result was consistent with the results found by Stewart et al; they found that the mean distance between the superior margin of the RWN to the OW was $2.7 \mathrm{~mm}$; and our results are also consistent with the results of Singla et al, who reported a mean of $2.19 \pm 0.43 \mathrm{~mm}$, ranging from 1.39 to $3.57 \mathrm{~mm}$, but were different from the measurements found by Paprocki et al on 10 cadaveric temporal bones; they reported the mean RW-OW distance to be $1.43 \pm 0.279 \mathrm{~mm}$; the possible reasons for this difference were attributed to the number of specimens analyzed. ${ }^{9,15,23}$

In the present study, the crista fenestra was evaluated only for its presence and its shape. The crista fenestra is a sharp bony crest, which occupies a considerable area of the circumference of the RWN; during cochlear implant surgery, it can be considered an obstacle to electrode insertion to the scala tympani.

B) Radiological and surgical study:

A HRCT scan was performed for the 20 cases subjected for cochlear implantation. The mean transverse diameter of the RWN was measured in the axial and coronal planes, and measured $1.27 \pm 0.4 \mathrm{~mm}$, while the height (the depth of the prechamber) was measured in the sagittal plane, and was $1.52 \pm 1.38 \mathrm{~mm}$. these measurements were consistent with the measurement taken from the cadaveric specimens $(1.18 \pm 0.25 \mathrm{~mm})$ for the maximum diameter of the niche, and which were $1.53 \pm 0.41 \mathrm{~mm}$ for the maximum height.

There was no significant difference between the average width and height measured radiologically and those measured on the temporal bone study, which ensures that the new imaging modalities are reliable methods that give an accurate data about anatomical measurements.

Our results were consistent with the results found by Veillon et al, who performed a radiological study using 75 temporal bones and found a RW transverse diameter ranging between 1.3 and $1.9 \mathrm{~mm}$; on the other hand it was completely different from the results of Takahashi et al, who measured the diameter and the height of the RW niche in only 5 specimens. $^{18,24}$

The radiological identification of the width and the height of the RW prechamber prior to surgery is essential in surgical planning regarding the type of implant, and the amount of niche drilling, if needed, as a very tight RW niche usually requires the use of slim electrodes, and a short RW prechamber (procident tegmen), will require minimal tegmen drilling to avoid going through a wrong scala or injuring the osseus spiral lamina.

\section{The Subcochlear Canaliculus (The Infracochlear Tunnel)}

Interestingly, to date, there are no published reports highlighting the surgical anatomy of the subcochlear canaliculus relevant for cochlear implantation, and to our knowledge this is the first work in the literature to do so.

Anatomically, the subcochlear canaliculus is a tunnel located between the fustis and the finiculus, and connects the RW prechamber with the petrous apex and, subsequently, the petrous carotid via a series of pneumatized air cells. Marchioni et al evaluated the endoscopic anatomy of this canaliculus in cases with cholesteatoma, and as a corridor for petrous apex lesions, they found it in only $84 \%$ of the 42 specimens in their study; endoscopically, they reported 
three different types of subcochlear canaliculus: Type A: represents a large tunnel to the petrous apex, detectable endoscopically; Type B: a small tunnel, with a connection to the petrous apex, not detectable endoscopically; Type $\mathbf{C}$ : the RW chamber is not connected to the petrous apex. ${ }^{25}$

In the current study, the lateral end of the infracochlear canaliculus was invisible in 2 out of the 20 (10\%) microscopically dissected cadaveric specimens; the finiculus bone merged medially with the fustis, and these tunnels were classified as type $C$ according to Marchioni. On the other hand, among the 20 cases subjected for cochlear implantation, the infracochlear tunnel was evaluated radiologically in the coronal and the sagittal views of the HRCT using the new OTOPLAN software. The tunnel was present in all cases in the study with variable degrees of pneumatization, and subsequently, we classified the subcochlear canaliculus radiologically into 4 types (-Fig. 2 ):

Type 1: Poorly pneumatized infracochlear air cell tract; limited subcochlear pneumatization. Noticed in only 3 cases.

Type 2: Well-pneumatized but not reaching the petrous apex in 6 cases.

Type 3: Well-pneumatized reaching the petrous apex, no connection with the petrous apex air cells in 9 cases.

Type 4: Well-pneumatized and connected to the petrous apex air cells was found only in 2 cases.

In our study, we consider the infracochlear air cell track a very common site and cause of misplacement of the electrode array not only in the hypotympanic air cells inferior to the cochlea, but also in the adjacent extracochlear sites, such as the petrous apex and the internal carotid canal. The configuration and the orientation of this track during a microscopic approach through posterior tympanotomy can be easily misinterpreted during surgery as a RW prechamber. The final position and the encountered resistance to insertion of the electrode, if inserted into this track, depend on the degree of its pneumatization. If the track is nonpneumatized or poorly pneumatized, the final position of the electrode array will be in the hypotympanic air cells and resistance to the insertion will be encountered; on the other hand, in well-pneumatized tracks, the electrode can reach the petrous apex and even the petrous carotid with minimal resistance. (-Fig. 6)

\section{The Direction of the Round Window Niche}

The boney walls of the RW prechamber start to develop by the $16^{\text {th }}$ week of the intrauterine life. The tegmen, the postis anterior (anterior pillar) and the postis posterior (posterior pillar) are the first to appear, while the fustis (inferior wall) is completely absent at this time. One week later, the fustis starts to develop, only reaching the anterior pillar by the $18^{\text {th }}$ week. By the $20^{\text {th }}$ week, the anterior and posterior pillar and the tegmen show a rapid increase in growth; the most intensive growth can be found in the anterior wall, where the inferior tympanic artery and the tympanic nerve run, which can be found as a boney canal parallel to the anterior pillar, followed by the tegmen (the superior wall). ${ }^{11}$

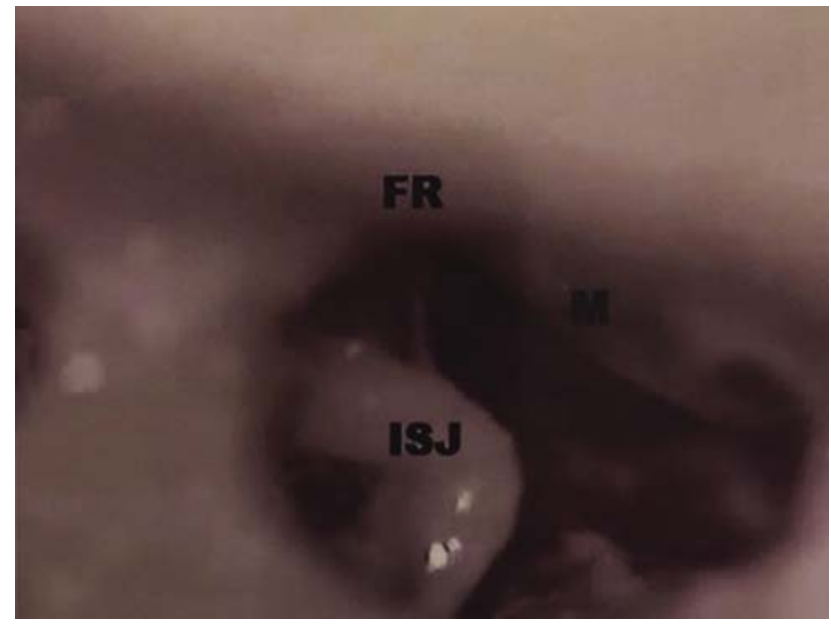

Fig. 6 The round window was not visible both thorough the facial recess and the external auditory canal.

The anterior pillar and tegmen of the round window prechamber begin to ossify as chondral bone, but the main parts of both will be finally formed by membranous bone. On the other hand, the posterior pillar ossification remains until the end of the development in the chondral bone ossification pattern. Membranous bones are rich in blood vessels and shows rapid growth, more than chondral bones, which results in a wide range of variability of the RW prechamber walls.

Depending on the rate of growth of the anterior pillar and the tegmen, the plane of the RWN or the direction at which it faces varies. In cases in which the anterior wall lengthens more than the tegmen, the RW will face posteriorly. When the tegmen becomes longer than the anterior wall, the direction of the RW will be inferior. The most common variant, when both of them grow in a proportionate way to each other, the RW prechamber will face the posteroinferior direction.

Owing to the impossibility to measure the lengthening of the tegmen, in the current study we measured the length of both the anterior and posterior pillar. The anterior pillar was measured from the most superior point or the dome of the tegmen until the junction with the fustis, the mean length of the postis anterior was $2.22 \pm 0.18 \mathrm{~mm}$, which was larger than the postis posterior, which was measured from the same point until it meets the fustis inferiorly, with a mean length of $2.09 \pm 0.20 \mathrm{~mm}$. The only limitation was the tremendous variations in the tegmen shape, and so the most superior point or its dome was taken as a reference for measurement.

There was a statistically significant difference between the mean difference between both pillars and the direction at which the niche faced. When both pillars were almost equal; the difference in the anterior pillar-posterior pillar length was $0.02 \pm 0.03$. In this occasion, there was no difficulty in the identification of the RW or in electrode insertion through the posterior tympanostomy, as the direction vector of insertion was directly to the scala tympani. This was found in 11 out of the 20 cases subjected to cochlear implantation in the current study. 
The direction of the RW was inferior in 7 cases; among those patients, the posterior pillar was significantly larger than the anterior, and the mean difference was $0.41 \pm 0.06 \mathrm{~mm}$. During the surgery, full visualization of the RW through the posterior tympanostomy was difficult despite progressive thinning of the posterior wall of the external auditory canal, and in two cases it was not visualized except through the external auditory canal after elevation of the tympanomeatal flap; however, we were able to insert the electrode through the RW after its visualization. In the situation of an inferiorly facing RW prechamber, misplacement of the electrode array can easily happen in an extracochlear site, mostly in the hypotympanic air cells, so a wider exposure and the identification of other anatomical landmarks, such as the OW or the stapedius tendon might be needed. (-Fig. 3, 4)

Lastly, a posteriorly-facing RWN was encountered only in two cases, with significant longer anterior pillar; the difference between both pillars was $0.61 \pm 0.01 \mathrm{~mm}$. During surgery, the RW was not completely visualized despite posterior tilting of the patient head and progressive thinning of the posterior wall of the FR until the mastoid segment of the facial nerve was clearly visible through a very thin covering bone. Among those two patients, we used the $30^{\circ}$ otoendoscope after meticulous elevation of the tympanomeatal flap for proper localization of the RW, and successful insertion of the electrode array was performed through a cochleostomy anteroinferior to the RW and not through the RW itself. In the situation of a posterior facing RW prechamber, the difficulty in visualization may not only lead to misplaced cochlear implant electrode, but also may end up in injury to the surrounding extracochlear structures during blind drilling along the cochlear curvature to the modiolus or to more catastrophic anterior drilling toward the internal carotid canal. (-Fig. 4)

\section{Conclusion}

Proper understanding of the topographic anatomy of the RW, including its direction of opening, and the distances from different adjacent structures in the tympanum, is essential for successful cochlear implantation surgery; it can help decision-making before surgery and is very useful to avoid many complications, such as misplacement into extracochlear sites and iatrogenic injury to the surrounding structures.

In our anatomical study, we were able to assess the parameters of relation of the round window to the surrounding structures in the tympanum; these parameters are more of cadaveric dissection limits than of real cases, because of the limited number of temporal bone specimens, which lacks age, racial and gender variations.

In the current surgical study, the anatomy of the subcochlear canaliculus was evaluated and was classified radiologically into four subtypes according to the pattern of its pneumatization. Lastly, the orientation of the RW, its direction, or at which direction it faces, was evaluated according to the relation between the different components comprising the RW prechamber (anterior, posterior pillars, and the tegmen).
The limitations in the present study were that the crista fenestra was evaluated only for its presence and its shape. This boney crest occupies a considerable area of the circumference of the RWN. Future studies have to be directed to the assessment of the relation between $\mathrm{RWh}, \mathrm{RWw}$, and the surface area and between the surface area and the shape of this boney crest among a bigger number of temporal bones, and in the presence of an accurate way for measurement. Also, the limited number of temporal bones was another limitation.

Conflict of Interests

The authors have no conflict of interests to declare.

\section{References}

1 Franz BK, Clark GM, Bloom DM. Surgical anatomy of the round window with special reference to cochlear implantation. J Laryngol Otol 1987;101(02):97-102

2 Proctor B, Bollobas B, Niparko JK. Anatomy of the round window niche. Ann Otol Rhinol Laryngol 1986;95(5 Pt 1):444-446

3 Clark GM. A surgical approach for a cochlear implant: an anatomical study. J Laryngol Otol 1975;89(01):9-15

4 Clark GM, Pyman BC, Bailey QR. The surgery for multiple-electrode cochlear implantations. J Laryngol Otol 1979;93(03):215-223

5 Burian K, Hochmair-Desoyer IJ, Hochmair ES. Hoeren uber ein cochlear implantat. Arch ONK-Heilk. 1981;231:569-570

6 Gantz BJ, Turner CW. Combining acoustic and electrical hearing. Laryngoscope 2003;113(10):1726-1730

7 Stidham KR, Roberson JB Jr. Cochlear hook anatomy: evaluation of the spatial relationship of the basal cochlear duct to middle ear landmarks. Acta Otolaryngol 1999;119(07):773-777

8 Erixon E, Högstorp H, Wadin K, Rask-Andersen H. Variational anatomy of the human cochlea: implications for cochlear implantation. Otol Neurotol 2009;30(01):14-22

9 Singla A, Sahni D, Gupta AK, Loukas M, Aggarwal A. Surgical anatomy of round window and its implications for cochlear implantation. Clin Anat 2014;27(03):331-336

10 Wysocki J. [Variability of the round window niche entry in children and adults]. Otolaryngol Pol 1998;52(04):463-466

11 Tóth M, Alpár A, Patonay L, Oláh I. Development and surgical anatomy of the round window niche. Ann Anat 2006;188(02):93-101

12 Hamamoto M, Murakami G, Kataura A. Topographical relationships among the facial nerve, chorda tympani nerve and round window with special reference to the approach route for cochlear implant surgery. Clin Anat 2000;13(04):251-256

13 Goravalingappa R. Cochlear implant electrode insertion: Jacobson's nerve, a useful anatomical landmark. Indian J Otolaryngol Head Neck Surg 2002;54(01):70-73

14 Aslan A. ãbrahim Tekdemir, Kõvanc G,nhan, G^rkem Eskiizmir, Alaittin Elhan, Anatomic Observations on Variations of the Round Window Niche and its Relationship to the Tympanic Membrane, Mediterr. J Otol 2006;2:52-57

15 Stewart TJ, Belal A. Surgical anatomy and pathology of the round window. Clin Otolaryngol Allied Sci 1981;6(01):45-62

16 Su WY, Marion MS, Hinojosa R, Matz GJ. Anatomical measurements of the cochlear aqueduct, round window membrane, round window niche, and facial recess. Laryngoscope 1982;92(05):483-486

17 Cohen D, Blinder G, Perez R, Raveh D. Standardized computed tomographic imaging and dimensions of the round-window niche. Int Tinnitus J 2005;11(02):158-162

18 Takahashi H, Sando I, Takagi A. Computer-aided three-dimensional reconstruction and measurement of the round window niche. Laryngoscope 1989;99(05):505-509 
19 Roland PS, Wright CG, Isaacson B. Cochlear implant electrode insertion: the round window revisited. Laryngoscope 2007;117 (08):1397-1402

20 Raine $\mathrm{CH}$, Hussain SS, Khan S, Setia RN. Anomaly of the facial nerve and cochlear implantation. Ann Otol Rhinol Laryngol Suppl 1995;166:430-431

21 Fayad JN, Wanna GB, Micheletto JN, Parisier SC. Facial nerve paralysis following cochlear implant surgery. Laryngoscope 2003;113(08):1344-1346

22 Duan JR, Luo HQ, Lu CJ, Wei JP, Li MZ, Xiong JP. [Round window area temporal bone section versus CT]. Zhonghua Er Bi Yan Hou Ke Za Zhi 2004;39(05):269-272
23 Paprocki A, Biskup B, Kozłowska K, Kuniszyk A, Bien D, Niemczyk $\mathrm{K}$. The topographical anatomy of the round window and related structures for the purpose of cochlear implant surgery. Folia Morphol (Warsz) 2004;63(03):309-312

24 FrancisV .SophieR, BogdanE, DanisiaH, MarieR; Imaging of the windows of the temporal bone. Seminars in Ultasound. CT and MRI 2001;22:271-280

25 Marchioni D, Alicandri-Ciufelli M, Piccinini A, Genovese E, Presutti L. Inferior retrotympanum revisited: an endoscopic anatomic study. Laryngoscope 2010;120(09):1880-1886 